Bio - grafía. Escritos sobre la Biología y su Enseñanza. ISSN 2027-1034

Edición Extraordinaria. p.p. 1520 - 1526

Memorias del IX Encuentro Nacional de Experiencias en Enseñanza de la Biología y la Educación Ambiental. IV Congreso Nacional de Investigación en Enseñanza de la Biología.

\title{
TALLER: RUTINAS DE PENSAMIENTO Y FORMACIÓN DE MAESTROS
}

\author{
Pedro Ramírez Sánchez ${ }^{1}$ \\ Yulieth Romero Rincón ${ }^{2}$ \\ Carlos Humberto Barreto Tovar ${ }^{3}$
}

\section{RESUMEN}

El propósito del taller es reconocer las rutinas de pensamiento como estrategias para la visibilización del pensamiento de los maestros en formación frente a cada uno de los componentes del Conocimiento Profesional del Profesor de Ciencias (CPPC).

El Conocimiento Profesional del Profesor de Ciencias (CPPC), según Valbuena (2007) y Bernal (2013) está conformado por mínimo cuatro componentes: Conocimiento Pedagógico (CP), Conocimiento Disciplinar (CD), Conocimiento Didáctico del Contenido (CDC) y Conocimiento Contextual (CC). Evidenciarlos en profesores en ejercicio (docentes expertos) es cuestión de posibilitar una visibilización de sus estructuras y concepciones de enseñanza y aprendizaje. Desde la aplicación de rutinas de pensamiento que tienen una intencionalidad marcada, permiten abordar, analizar y discutir dentro de un marco de planeación cuáles elementos predominan, si están ausentes o si son incipientes desde la experticia o el grado de formación. En este orden de ideas, seleccionar algunas rutinas requiere de dos elementos; uno, la planeación del docente formador que incluye entre otros, las metas de comprensión y dos, la intencionalidad y alcance de la misma rutina.

En este taller, se desarrollarán las rutinas de pensamiento "Generar, Clasificar, Conectar y Elaborar (GCCE)" está pensada para la construcción de mapas conceptuales que evidencien modelos mentales (Ritchhart, 2015) desde la capacidad de síntesis y relación de ideas; y la rutina de pensamiento denominada" Antes Pensaba - Ahora Pienso", la cual conduce a una revisión de ideas previas y al establecimiento de contrastes en la adquisición de nuevas concepciones. Éstas dos rutinas conducen principalmente a comprobar el alcance de logro de la meta de contenido y facilitan los elementos de retroalimentación.

PALABRAS CLAVES: pensamiento, enseñanza para la comprensión, conocimiento profesional del profesor de ciencias, estrategias de enseñanza, rutinas de pensamiento.

\footnotetext{
${ }^{1}$ Universidad de La Sabana. Facultad de Educación. Maestría en Pedagogía. Correo: pedrorasa@unisabana.edu.co

2 Universidad de La Sabana. Facultad de Educación. Maestría en Pedagogía. Correo: yuliethrori@unisabana.edu.co

3 Universidad de La Sabana. Facultad de Educación. Maestría en Pedagogía. Correo: carlos.barreto2@unisabana.edu.co
} 
Bio - grafía. Escritos sobre la Biología y su Enseñanza. ISSN 2027-1034

Edición Extraordinaria. p.p. 1520 - 1526

Memorias del IX Encuentro Nacional de Experiencias en Enseñanza de la Biología y la Educación Ambiental. IV Congreso Nacional de Investigación en Enseñanza de la Biología.

\section{ABSTRACT}

The purpose of the workshop is to recognize the thought routines as strategies for the visibilization of the thinking of the teachers in formation in front of each of the components of the Professional Knowledge of the Professor of Sciences (CPPC).

According to Valbuena (2007) and Bernal (2013), the Professional Knowledge of the Professor of Sciences (CPPC) consists of at least four components: Pedagogical Knowledge (CP), Disciplinary Knowledge (CD), Contextual Knowledge (DC). It is a question of making them visible in professors with experience (teachers who are experts) in order to make their structures and conceptions of teaching and learning possible. From the application of thought routines that have a marked intentionality, allow to approach, analyze and discuss within a planning framework which elements predominate, if they are absent or if they are incipient from the expertise or the degree of training. In this vein, selecting some routines requires two elements; One, the planning of the teacher trainer that includes among others, the goals of understanding and two, the intentionality and scope of the same routine.

In this workshop, the "Generate, Classify, Connect and Develop (GCCE)" thought routines will be developed for the construction of conceptual maps that evidence mental models (Ritchhart, 2015) from the capacity of synthesis and relation of ideas; And the thinking routine called "Before I Thought - Now I Think", which leads to a review of previous ideas and the establishment of contrasts in the acquisition of new conceptions. These two routines mainly lead to check the achievement scope of the content goal and facilitate the feedback elements.

KEYWORDS: Thinking, teaching for understanding, professional knowledge of the science teacher, teaching strategies, thinking routines.

\section{INTRODUCCIÓN}

Desde el énfasis de Docencia para el desarrollo del pensamiento científico y tecnológico de la Maestría en Pedagogía de la Universidad de La Sabana se asume al maestro como un profesional reflexivo, el cual se cuestiona frente a lo que sucede cotidianamente en el aula. De allí que desde el ejercicio propio de los formadores de formadores se busque la implementación de diversas estrategias y metodologías que permitan visibilizar y reconocer el pensamiento de los maestros en formación posgradual de forma que permita un ciclo de reflexión propio conducente a enriquecer sus prácticas de enseñanza.

Es necesario preguntarse si podemos conocer el razonamiento argumentativo del alumnado y los procesos que tienen lugar en su mente. En opinión de Kuhn (1992), el diálogo argumentativo exterioriza el razonamiento argumentativo: "Es decir, no hay forma de conocer exactamente lo que ocurre en el interior de la mente, pero una de las formas en que podemos aproximarnos es prestando atención a las discusiones entre estudiantes sobre cuestiones de ciencias". Así es interesante el argumento que busca favorecer el aprendizaje de destrezas de pensamiento obviando el hecho de que el aprendizaje escolar es de carácter disciplinar (García, 1997) y que por lo tanto, son relevantes, junto con los contenidos, los propósitos, los métodos y las formas de 
Bio - grafía. Escritos sobre la Biología y su Enseñanza. ISSN 2027-1034

Edición Extraordinaria. p.p. 1520 - 1526

Memorias del IX Encuentro Nacional de Experiencias en Enseñanza de la Biología y la Educación Ambiental. IV Congreso Nacional de Investigación en Enseñanza de la Biología.

comunicación de la disciplina (Perkins, 1999). La adquisición y apropiación de estas destrezas se realiza, finalmente, a partir de una disciplina concreta, desde su lógica interna, de sus contenidos construidos con unos ciertos métodos y ciertos propósitos.

En este orden de ideas, el desarrollo del pensamiento cuyo propósito en la escuela es permitir que el sujeto se prepare para reflexionar sobre su propio aprendizaje y logre vincular activamente sus conocimientos, saberes y experiencias en la solución de problemas de la vida diaria. Para ello, es as í como en los últimos años se ha venido consolidando la cultura de pensamiento, al asumirla como "un ámbito del aula en el que varias fuerzas (lenguaje, valores, expectativas y hábitos) operan conjuntamente para expresar y reforzar... el buen pensamiento" (Tishman, Perkins \& Jay, 2006).

En esa línea las fuerzas culturales (tabla 1) proporcionan puntos de influencia para crear una cultura de pensamiento y pueden ayudar a comprender el contexto en el que se pueda usar las rutinas de pensamiento y otras formas de hacer visible el pensamiento.

\begin{tabular}{|l|l|}
\hline Fuerza Cultural & \multicolumn{1}{|c|}{ Concepción general } \\
\hline Expectativa & $\begin{array}{l}\text { Expectativa para el aprendizaje. Ofrecen el enfoque y la dirección } \\
\text { para el pensamiento. Incluyen las metas y los propósitos del } \\
\text { grupo. Establecer conexiones y sintetizar pensamiento. }\end{array}$ \\
\hline Oportunidades & $\begin{array}{l}\text { Son el mecanismo mediante el cual se pueden realizar las } \\
\text { expectativas. Un contenido desafiante, provocador y útil. } \\
\text { Interacción significativa con el contenido. Construcción de } \\
\text { experiencias, conexión entre ideas, }\end{array}$ \\
\hline Tiempo & $\begin{array}{l}\text { Dar tiempo para pensar en el aula, en el grupo, en espacios } \\
\text { adicionales. El buen pensamiento requiere tiempo, es decir, } \\
\text { desarrollo de ideas, conexiones y comprensiones. Se logra con el } \\
\text { uso de rutinas como estructuras comunes de discusión, brindando } \\
\text { autonomía y eficiencia. }\end{array}$ \\
\hline Modelar & $\begin{array}{l}\text { El docente muestra a los estudiantes como hacer algo, un } \\
\text { proceso, una tarea, un trabajo. El docente debe ser pensador y } \\
\text { aprendiz, participativo de las discusiones, cercano en el trabajo de } \\
\text { cada grupo. }\end{array}$ \\
\hline Ambiente Físico & $\begin{array}{l}\text { Los docentes nombran, detectan y destacan el pensamiento y las } \\
\text { ideas que son importantes dentro del contexto de aprendizaje. } \\
\text { Cambio de un lenguaje individual absoluto a un lenguaje } \\
\text { condicional que reconoce posibilidades, alternativas y } \\
\text { perspectivas. }\end{array}$ \\
\hline $\begin{array}{l}\text { iQué dice el aula desde su disposición, cómo se espera la } \\
\text { interacción entre los estudiantes, donde está el escritorio del } \\
\text { docente, que hay en las paredes, quien puso información o algo } \\
\text { allí, que comunica un aula que no tiene nada en las paredes? El } \\
\text { ser humano está continuamente construyendo y reconstruyendo el } \\
\text { medio ambiente para satisfacer las necesidades.LA disposición de }\end{array}$ \\
\hline
\end{tabular}


Bio - grafía. Escritos sobre la Biología y su Enseñanza. ISSN 2027-1034

Edición Extraordinaria. p.p. 1520 - 1526

Memorias del IX Encuentro Nacional de Experiencias en Enseñanza de la Biología y la Educación Ambiental. IV Congreso Nacional de Investigación en Enseñanza de la Biología.

\begin{tabular}{|c|c|}
\hline & $\begin{array}{l}\text { los resultados en forma de galería es una posibilidad de dialogar, } \\
\text { construir, conectar. }\end{array}$ \\
\hline Interacciones & $\begin{array}{l}\text { Importancia de preguntar y escuchar, interés por el pensamiento } \\
\text { de estudiante, búsqueda de colaboración significativa. La } \\
\text { comprensión y el aprendizaje profundo se alcanza al escuchar y } \\
\text { tomar las ideas y puntos de vista del otro, también presentar los } \\
\text { pensamientos propios ante otro, mejor aún apoyado en evidencias } \\
\text { y razones. Esta habilidad requiere de una retroalimentación } \\
\text { constante. }\end{array}$ \\
\hline Rutinas & $\begin{array}{l}\text { Están diseñadas para escalonar y apoyar el pensamiento y con el } \\
\text { tiempo conseguir un sentido de independencia al comprometer a } \\
\text { los estudiantes con el pensamiento. }\end{array}$ \\
\hline
\end{tabular}

Tabla 1. Fuerzas culturales. Adaptación propia de Ritchhart (2015), pg. 329 - 339.

En esta transformación educativa se reconocen diferentes fuerzas culturales, en las que se encuentran las rutinas de pensamiento, que han demostrado su pertinencia dentro de la formación de maestros para el desarrollo del pensamiento científico y tecnológico, además de fortalecer la controversia argumentada entre otras. Las rutinas de pensamiento se constituyen como "procedimientos sencillos, que por lo general cuentan con pocos pasos, ofrecen un marco para enfocar la atención en movimientos específicos de pensamiento que ayudan a construir la comprensión" (Ritchhart, Church \& Morrison, 2014). Según Ritchhart, Church \& Morrison (2014) las rutinas de pensamiento pueden ser entendidas como herramientas, estructuras o patrones de comportamiento que al ser apropiados dentro del aula posibilitan la vivencia de una cultura del pensamiento, son flexibles y pueden ser adaptadas a diferentes contextos educativos, puesto que es el maestro quien da la intencionalidad pedagógica a su implementación.

De igual manera desde las metas y desempeños de comprensión que se establezcan en una planeación estas rutinas pueden agruparse en tres categorías, la primera de ellas para presentar y explorar ideas, la segunda para sintetizarlas y organizarlas y la tercera categoría corresponde a aquellas rutinas orientadas a profundizar.

Teniendo en cuenta el marco anterior, dentro de la Maestría en Pedagogía, específicamente el énfasis docencia para el desarrollo del pensamiento científico y tecnológico, se han venido implementando rutinas de pensamiento tales como: antes pensaba, ahora pienso, generar, clasificar conectar y elaborar (GCCE) y conexiones, desafíos, conceptos y cambios (CDCC), rutinas que se encuentran dentro de la categoría de sintetizar y organizar ideas. Con estas rutinas se ha buscado que los estudiantes puedan reflexionar frente a referentes conceptuales propios del énfasis, logrando establecer relaciones entre la teoría y la práctica pedagógica.

Las rutinas de pensamiento se han implementado dentro del enfoque de la enseñanza para la comprensión (Stone et al., 1999), el cual presenta elementos estructurales claves como los hilos conductores, el tópico generador, las metas de comprensión, los desempeños de comprensión y la valoración continua, todos orientados a lograr la comprensión entendida como "la habilidad de pensar y actuar con flexibilidad a partir 
Bio - grafía. Escritos sobre la Biología y su Enseñanza. ISSN 2027-1034

Edición Extraordinaria. p.p. 1520 - 1526

Memorias del IX Encuentro Nacional de Experiencias en Enseñanza de la Biología y la Educación Ambiental. IV Congreso Nacional de Investigación en Enseñanza de la Biología.

de lo que uno sabe. Para decirlo de otra manera, la comprensión de un tópico es la capacidad de desempeño flexible con énfasis en la flexibilidad" (Stone et al., 1999 p.4)

En este taller se implementarán diferentes rutinas de pensamiento, en las cuales se profundizará (tabla 2) a continuación partiendo de lo establecido por Ritchhart, Church \& Morrison (2014).

\begin{tabular}{|l|l|l|}
\hline \multicolumn{2}{|c|}{ RUTINAS PARA SINTETIZAR Y ORGANIZAR IDEAS } \\
\hline Nombre de la rutina & $\begin{array}{l}\text { Movimientos claves } \\
\text { del pensamiento }\end{array}$ & Notas y descripción \\
\hline $\begin{array}{l}\text { Generar - Clasificar - } \\
\text { Conectar - Elaborar: } \\
\text { GCCE }\end{array}$ & $\begin{array}{l}\text { Descubrir y organizar } \\
\text { conocimiento previ } \\
\text { para identificar } \\
\text { conexiones }\end{array}$ & $\begin{array}{l}\text { Resalta los pasos de pensamiento } \\
\text { para hacer un mapa conceptual } \\
\text { efectivo que organice y revele el } \\
\text { pensamiento }\end{array}$ \\
\hline $\begin{array}{l}\text { Antes Pensaba - Ahora } \\
\text { Pienso }\end{array}$ & $\begin{array}{l}\text { Reflexión } \\
\text { Metacognición }\end{array}$ & $\begin{array}{l}\text { Se usa para ayudar a los } \\
\text { aprendices a reflexionar sobre } \\
\text { como su pensamiento ha } \\
\text { cambiado a lo largo del tiempo }\end{array}$ \\
\hline $\begin{array}{l}\text { Conexiones - Desafíos } \\
\text { Conceptos - cambios: } \\
\text { CDCC }\end{array}$ & $\begin{array}{l}\text { Establecer } \\
\text { conexiones, } \\
\text { identificar el concepto } \\
\text { clave, cuestionar y y } \\
\text { considerar } \\
\text { implicaciones }\end{array}$ & $\begin{array}{l}\text { Rutina que ayuda a identificar los } \\
\text { puntos claves de un texto } \\
\text { complejo para discutir, se requiere } \\
\text { de buen texto, libro o } \\
\text { documento. }\end{array}$ \\
\hline
\end{tabular}

Tabla 2. Adaptación propia de Ritchhart (2015), Pg. 94-96.

\section{PROPÓSTIOS DEL TALLER}

- Socializar elementos propios de las fuerzas culturales para el desarrollo del pensamiento desde la implementación de rutinas.

- Reconocer las rutinas de pensamiento como estructuras de la clase que posibilitan la construcción colectiva y colaborativa del conocimiento.

- Proponer un diálogo de saberes entre pares académicos que permita establecer la relación entre rutinas de pensamiento, estrategia de enseñanza, proceso de formación docente y construcción colectiva de pensamiento. 
Bio - grafía. Escritos sobre la Biología y su Enseñanza. ISSN 2027-1034

Edición Extraordinaria. p.p. 1520 - 1526

Memorias del IX Encuentro Nacional de Experiencias en Enseñanza de la Biología y la Educación Ambiental. IV Congreso Nacional de Investigación en Enseñanza de la Biología.

\section{ASPECTOS PROCEDIMENT ALES}

Momentos del taller:

\section{Inicio}

Tiempo: 15 minutos

Materiales: papel craf, cinta, marcadores, post it.

Implementación de la primera parte de la rutina de pensamiento Antes pensaba... ahora pienso a partir de las siguientes preguntas orientadoras:

¿Qué entiende por conocimiento profesional del profesor?

¿Qué componentes incluiría el conocimiento profesional del profesor?

¿Cómo puede visibilizarse el pensamiento del profesor de ciencias?

¿Cómo promovería una cultura de pensamiento desde su ejercicio docente?

Socialización de elementos conceptuales generales frente a cultura de pensamiento, enseñanza para la compresión y rutinas de pensamiento.

\section{Desarrollo}

Tiempo: $25-30$ minutos

Materiales: fotocopias de lectura, marcadores, hojas blancas y de colores, tijeras, cinta y papel craf.

- Implementación de las rutinas de pensamiento a partir de lecturas o videos cortos:

- Generar-Clasificar- Conectar-Elaborar

- Conexiones - Desafíos - Conceptos - Cambios

- Organización del grupo en dos subgrupos de 10 a 15 personas.

- Lectura individual de cinco minutos.

\begin{tabular}{|c|c|}
\hline Rutina GCCE & Rutina CDCC \\
\hline $\begin{array}{l}\text { Escribir un listado de conceptos de la } \\
\text { lectura. } \\
\text { Identificar los conceptos principales y } \\
\text { secundarios. }\end{array}$ & $\begin{array}{l}\text { Responder las preguntas orientadoras. } \\
\text { Registrar preguntas propias que le haría } \\
\text { al texto o definir elementos con los que no } \\
\text { está de acuerdo. }\end{array}$ \\
\hline $\begin{array}{l}\text { Pegar los conceptos seleccionados en } \\
\text { relación al concepto principal dado. }\end{array}$ & $\begin{array}{l}\text { Seleccionar los conceptos centrales de la } \\
\text { lectura. }\end{array}$ \\
\hline $\begin{array}{l}\text { Construya una definición amplia de las } \\
\text { relaciones que encuentre en un solo } \\
\text { párrafo. }\end{array}$ & $\begin{array}{l}\text { Definir los cambios para su práctica } \\
\text { pedagógica. }\end{array}$ \\
\hline
\end{tabular}


Bio - grafía. Escritos sobre la Biología y su Enseñanza. ISSN 2027-1034

Edición Extraordinaria. p.p. 1520 - 1526

Memorias del IX Encuentro Nacional de Experiencias en Enseñanza de la Biología y la Educación Ambiental. IV Congreso Nacional de Investigación en Enseñanza de la Biología.

Momento de galería para observación de la construcción colectiva.

\section{Cierre}

- Puesta en común y diálogo de saberes frente a la aplicación de las rutinas y sus posibilidades dentro de la formación de maestros.

- Implementación de la segunda parte de la rutina de pensamiento Antes pensaba... ahora pienso desde las preguntas orientadoras iniciales.

- Retroalimentación cálida y fría.

\section{BIBLIOGRAFÍA}

- Kuhn, D. (1992). Thinking as argument. Harvard Educational Review, 62(2), 155-179.Kuhn

- Martínez R, C. A., \& Valbuena, E. (2013). El conocimiento profesional de los profesores de ciencias sobre el conocimiento escolar: resultados de investigación. Bogotá: Universidad Distrital Francisco José de Caldas.

- Ritchhart, R; Church, M; Morrison, K. (2014). Hacer visible el pensamiento: Cómo promover el compromiso, la comprensión y la autonomía de los estudiantes. Paidós. Traducido por: María Barrera y Patricia León. Título original: Making Thinking Visible.

- Stone et al., (1999). La enseñanza para la comprensión: Vinculación entre la investigación y la práctica. Buenos Aires: Paidós.

- Tishman, Perkins \& Jay. (2006). Un aula para pensar: Aprender y enseñar en una cultura de pensamiento. Buenos Aires: Aique.

- Valbuena, E. O. (2007). Memorias de trabajo de Grado. Madrid - España: Universidad Complutense de Madrid. 